\title{
Using IT in the undergraduate classroom: should we replace the blackboard with PowerPoint?
}

\author{
Attila Szabo $^{\mathrm{a}, *}$, Nigel Hastings ${ }^{\mathrm{b}}$ \\ ${ }^{a}$ Department of Life Sciences, The Nottingham Trent University, Clifton Lane, Nottingham NG11 8NS, UK \\ ${ }^{\mathrm{b}}$ Department of Primary Education, The Nottingham Trent University, Clifton Lane, Nottingham NG11 8NS, UK
}

\begin{abstract}
Three studies were performed to investigate the efficacy of digital PowerPoint lecturing in undergraduate classrooms. In the first study, students' opinion about PowerPoint lectures was surveyed after receiving all their lectures in one module in PowerPoint. Grades of one cohort were then compared with the grades of another taking the same test one year earlier. No significant differences were found. In another study, students received a mock test 1 week following: (1) an overhead lecture, (2) a PowerPoint lecture and (3) a PowerPoint lecture with lecture notes. There were no significant differences between the two PowerPoint lectures both of which resulted in higher grades than the overhead lecture. In the third study, two cohorts had two identical lectures, in a counterbalanced order, presented either with PowerPoint or by using overheads. The results revealed that the lecture difficulty, but not the method of lecturing, contributed to the grade differences on two mock tests. It is suggested that the efficacy of PowerPoint lecturing may be case specific rather than universal. (C) 2000 Elsevier Science Ltd. All rights reserved.
\end{abstract}

Keywords: Classroom; IT; Lectures; Overhead; PowerPoint

\section{Introduction}

The PowerPoint software, included in the 'Microsoft Office' package is a powerful presentation tool. Nowadays it has replaced the traditionally used colour slides and overheads

\footnotetext{
* Corresponding author. Tel.: +44-115-848-3362.

E-mail address: attila.szabo@ntu.ac.uk (A. Szabo).
}

0360-1315/00/\$ - see front matter (C) 2000 Elsevier Science Ltd. All rights reserved.

PII: S0360-1315(00)00030-0 
at important conferences. Originally PowerPoint was developed for commercial and business purposes, but it has quickly penetrated the scientific and educational circles as well. PowerPoint is a user-friendly package that can be used for the creation of visually clear, dynamic and attention capturing presentations (Holzl, 1997). Its adoption compels the speaker into a well-organised path on which the most important points are emphasised (Harknett \& Cobane, 1997). Its use in education has been already pioneered at many Universities and Colleges around the world. Lecturers are warned, however, that their true challenge is to use PowerPoint, and IT in general, for the enrichment of students' learning rather than simply for the improvement and/or modernisation of their performance in the classroom (Sipress, 1995).

\section{Literature review}

To date, the empirical evaluation of PowerPoint assisted lecturing in higher education is limited. Education databases, as BIDS and ERIC, do not contain any results-based documents giving relevant information. In a wider literature search, only three research reports could be traced. The first presents the findings from a pilot study, conducted in the US, and it is only available on an Internet site (Evans, 1998). In this study, 161 students who were enrolled in a General Psychology module, were tested. The module was offered to two groups. One group $(n=87)$ received traditional lectures delivered with overhead transparencies. The other group $(n=64)$ received the lectures with the aid of the PowerPoint software. At the end of the semester, the mean grade on all examinations was greater for the PowerPoint group than for the overhead lecture group (81.6 versus $76.9 \%$ ). Furthermore, the total absences were less in the former than in the latter group (2.1 versus $3.7 \%)$. The researcher also noticed that in the PowerPoint group some disruptive behaviour, such as inappropriate remarks, hostile or obnoxious attitudes and overt expression of boredom, occurred less frequently. These findings appear to be promising because they suggest that lectures delivered in electronic format using the PowerPoint software will: (1) increase students' grades, (2) improve lecture attendance rates, and (3) may reduce certain disruptive behaviours during the lectures.

Harknett and Cobane (1997) provide another, but mainly descriptive, account of the efficacy of PowerPoint in lecturing. Eighty percent of the students surveyed by the authors felt that PowerPoint lectures benefited their learning. Some felt that the visual emphasis in PowerPoint helped them recall the lecture material at the time of examination. However, apart from the students' positive attitude towards this method of lecturing, no other significant benefits of PowerPoint were disclosed. The authors suggest that PowerPoint slides are easy to update and that they can provide excellent opportunity for creating electronic handouts. These handouts can be linked to World Wide Web (WWW) pages from where the students can download them, print them, or revisit them if they need to. However, the findings emerging from this study cannot be generalised because it is unclear how many students were surveyed and how many lectures were presented with PowerPoint.

The third, and the most recent, account on PowerPoint lecturing is presented by Lowry (1999). In this inquiry three cohorts of students $(n>130$ in each), enrolled in an Environmental Science course in the UK, were tested. The first cohort (1994/95) received the lectures in the traditional way, using overhead transparencies. The second (1995/96) and the 
third (1996/97) cohorts received the lectures with PowerPoint. All students were assessed with a 30-item multiple-choice test. However, the three cohorts did not receive the same test, although the format was identical. The results revealed that the two PowerPoint lecture groups achieved better grades than the traditional-lecture cohort (51.8 and 51.9\% versus $43.5 \%$ ). Further, students exposed to PowerPoint lecturing had a positive attitude towards the method. Some of the features that the 86 students in the 1996/97 cohort found specially appealing were: the use of a PC (43\%), the visual aids $(22 \%)$, presentation format $(16 \%)$, lecture structure $(16 \%)$ and clarity $(12 \%)$. With the condition that some essential assumptions are met, (Lowry, 1999) study provides evidence for the benefits of PowerPoint lecturing on students' performance in higher education. These assumptions, which should be tested in future studies whenever possible, include: (1) the student cohorts are drawn from the same population, (2) the lecturing style and the lecture content are consistent, and (3) the assessment levels are comparable.

Based on the limited but promising evidence emerging from these few studies on PowerPoint lecturing, three inquiries were undertaken to evaluate further, the efficacy of this lecturing method in the higher education. The first study aimed to examine three things. First, how the students in a Sports Science course at The Nottingham Trent University feel about PowerPoint lecturing. The second aim of this study was to identify what students would like to see in future PowerPoint lectures. Finally, the third goal of the first study was to determine, whether this method of content delivery benefits their first in-class test-performance at the university. In the second inquiry, students' performance on three mock-tests following lectures using overheads, a digital PowerPoint presentation and a digital PowerPoint presentation combined with the handing out of all the lecture notes were compared. Finally, in the third study, the possible mediating effects of the lecture difficulty and students' preparation were examined. In this inquiry, two groups received two identical lectures in a counterbalanced order, one using PowerPoint and one using overheads only, 1 week apart.

\section{Study I}

\subsection{Rationale and hypothesis}

Some unique elements involved in PowerPoint lecturing could be expected to stimulate attention (Lowry, 1999). These elements include colour, pacing through line-by-line or conceptby-concept presentation of the information, flexibility for graphical interfacing, a well thought pre-organisation and easy variation of the size and the type of the fonts (Harknett \& Cobane, 1997; Holzl, 1997). Longer and deeper attention can benefit students' learning as well as the lecturing atmosphere by reducing distraction (Evans, 1998). Therefore, the replacement of the blackboards and overhead projectors with PowerPoint lectures was expected to be beneficial for teaching and learning. The present study surveyed students' opinion in a Sport Science and Administration course about PowerPoint-based lectures in contrast to the traditional lectures. The operational definition for 'traditional lectures' in the context of the current study was: "Lectures delivered without the significant use of IT equipment other than overhead projectors and possibly the occasional use of an audio-visual (VHS and audio playback) apparatus." PowerPoint lectures were conceptualised as: "Lectures in which the content, and 
complementary diagrams or pictures, is presented electronically with the aid of the PowerPoint software. This is done with little or no reliance (unless it is necessary for the sake of the clarification of students' questions) on overhead projection or blackboard usage." The following two hypotheses were tested:

1. Students will prefer PowerPoint lectures in contrast to traditional lectures.

2. After four PowerPoint lectures students will achieve better grades, on their first test at the university, than after four traditional lectures.

\subsection{Method}

One hundred and fifty-five male and female students participated in this study. Students enrolled in the first $(n=50)$ and the third year $(n=51)$ of their course have received all their lectures, in one of the modules, in PowerPoint format during the first semester of the academic year 1998/99. These modules were 'Motor Learning' for the first year students and 'Strength, Power and Endurance in Sport and Exercise' for the third year students. A group of second year students $(n=54)$ received one lecture, in 'Social Psychology of Sport and Exercise', in PowerPoint to see whether opinions differ among those who are habituated to the method and those who experience it as a new and isolated out of the ordinary experience.

A Hi-Grade notebook computer and a Viglen PC, equipped with versions 7.0 and 4.0, respectively, of the PowerPoint software were used along with either a VGA portable or a fixed digital projector. A ten-item questionnaire was designed and used to measure how the students feel about PowerPoint lecturing in contrast to traditional lectures. The 10 items were selected on the basis of the key elements of PowerPoint that were cited in the literature as possibly beneficial for learning (Evans, 1998; Holzl, 1997; Lowry, 1999). These elements are: attention capturing and maintenance, visual clarity, clear emphasis on key concepts, stimulation of interest, motivation to attend lectures, organisation of the delivery of the lectures, proper pacing of the delivery of the lectures, and understanding of the lecture material. A last question asked students whether they believed that PowerPoint lecturing is beneficial to their learning. Another ten-item questionnaire was used to identify those aspects of PowerPoint slides that are the most appreciated by the students. This measure was taken with the intention of using the answers in the design of future PowerPoint lectures. The two questionnaires were presented on the same form after the students in years one and three have attended at least 10 lectures in which the material was delivered with the aid of the PowerPoint software. For the second year students, the questionnaires were presented immediately after their only experience of a PowerPoint lecture.

The questionnaires were given to all participants with computer-readable answer cards to facilitate later scoring. Students could complete the questionnaires anonymously if they so desired. The questions were rated on a five-point agreement-disagreement scale that ranged from strongly agree to strongly disagree with a middle score of three indicating 'uncertainty'. The first questionnaire was used for comparing students' opinion about PowerPoint lectures in contrast to traditional lectures and was constructed with alternating positive and negative statements to avoid directional bias. For example, in the first question students were asked to 
indicate whether PowerPoint lectures are more attention capturing than traditional lectures (positive direction). Then, in the second question they were asked to indicate whether PowerPoint lectures are less interesting than the traditional lectures (negative direction). The negative items were reversed for scoring.

The second questionnaire was not used for comparison purposes. It was simply devised to assess the most popular features of the PowerPoint lectures. The 10 items on each questionnaire are listed along with the obtained answers in Tables 1 and 2.

Finally, to compare the perceived and the actual benefits of PowerPoint lecturing on learning performance, the first year students' grades on the first in-class test were compared to the grades obtained by students taking the same test in the same module in the previous year. This test was the first test written by these students at the university in both academic years. The lecture material and the test questions were the same for the two cohorts. Regrettably, a similar comparison could not be done for the third year students, because their module was offered for the first time in 1998/99.

\subsection{Results and discussion}

Given the descriptive and exploratory nature of the study, only percentages were calculated for the various answers. To obtain a preference (for PowerPoint) score the agree and strongly agree scores were combined. To generate a dislike (for PowerPoint) score the same principle was applied for the disagree and strongly disagree scores. As seen in Table 1, over $90 \%$ of the students in the three cohorts believed that the new method is more attention capturing than the traditional method of lecturing. This trend in answers supports the theoretical arguments presented for the utility of PowerPoint in lecturing (Harknett \& Cobane, 1997; Lowry, 1999). In light of such arguments, it is possible that some of the flexible features of PowerPoint, such as colour, variation in font, image, dynamic model building, or multimedia capabilities attract and/or maintain the students' attention. The students' enthusiasm for digital lecturing may also be due to the fact that PowerPoint lectures are better structured, (or were better structured in this study) hence the instructor could follow a well-organised path. However, it is also possible that PowerPoint lectures offer an 'out-of-ordinary' or novel experience to students. In this case, the attention capturing power of the method could be expected to fade away once the novelty effect disappears.

Parallel to the attention capturing power of the method, over $85 \%$ of the three cohorts confirmed that PowerPoint lectures are more interesting than traditional lectures. As with attention capturing power, it is hard to establish whether the method's features, its novelty, or both are the underlying cause for the pattern of these answers. The visual clarity and the emphasis on important concepts were also seen as an advantage for the method. However, the method was not so well praised when the pacing of the presentation for lecture note taking was considered. Such a trend in answers could be expected because the speed of the presentation, in relation to the amount of information projected, is independent of the method.

Over $65 \%$ of the students felt that the method is motivating them to come to the lectures. These affirmations support the findings of Evans (1998) that PowerPoint lectures help in increasing attendance rate at lectures. A better structuring of the lectures was affirmed by $82 \%$ of the students in the three cohorts, which, along with the greater attention paid to lectures, 
Table 1

Students were asked to compare PowerPoint lectures to traditional overhead or blackboard assisted lectures. The numbers represent percentages

\begin{tabular}{|c|c|c|c|c|c|c|c|c|c|}
\hline Question & $\begin{array}{l}\text { Agree } \\
\text { (Year 1) }\end{array}$ & $\begin{array}{l}\text { Agree } \\
\text { (Year 2) }\end{array}$ & $\begin{array}{l}\text { Agree } \\
\text { (Year 3) }\end{array}$ & $\begin{array}{l}\text { Uncertain } \\
\text { (Year 1) }\end{array}$ & $\begin{array}{l}\text { Uncertain } \\
\text { (Year 2) }\end{array}$ & $\begin{array}{l}\text { Uncertain } \\
\text { (Year 3) }\end{array}$ & $\begin{array}{l}\text { Disagree } \\
\text { (Year 1) }\end{array}$ & $\begin{array}{l}\text { Disagree } \\
\text { (Year 2) }\end{array}$ & $\begin{array}{l}\text { Disagree } \\
\text { (Year 3) }\end{array}$ \\
\hline Are more attention capturing & 92.0 & 90.8 & 90.2 & 6.0 & 7.4 & 7.8 & 2.0 & 1.9 & 2.0 \\
\hline Are more $\left(\right.$ less) ${ }^{\mathrm{a}}$ interesting & 86.0 & 90.6 & 88.4 & 12.0 & 7.5 & 13.7 & 2.0 & 1.9 & 7.9 \\
\hline $\begin{array}{l}\text { Are easier to follow and/or to } \\
\text { understand }\end{array}$ & 81.2 & 68.0 & 84.4 & 14.6 & 18.9 & 9.8 & 4.2 & 13.2 & 5.9 \\
\hline Are visually clearer (less clear) ${ }^{\mathrm{a}}$ & 96.0 & 88.9 & 76.5 & 2.0 & 9.3 & 7.8 & 2.0 & 1.9 & 15.7 \\
\hline $\begin{array}{l}\text { Better emphasize the important } \\
\text { concepts }\end{array}$ & 91.8 & 92.5 & 84.4 & 8.2 & 3.8 & 13.7 & 0.0 & 3.8 & 2.0 \\
\hline $\begin{array}{l}\text { Are better (less well) }{ }^{\mathrm{a}} \text { paced with note- } \\
\text { taking }\end{array}$ & 44.0 & 79.2 & 62.7 & 28.0 & 11.3 & 15.7 & 26.0 & 9.5 & 21.6 \\
\hline $\begin{array}{l}\text { Maintain focus on the presentation } \\
\text { for a longer time }\end{array}$ & 46.0 & 68.5 & 49.0 & 46.0 & 24.1 & 39.2 & 8.0 & 7.4 & 11.8 \\
\hline $\begin{array}{l}\text { Are more (less) }{ }^{\mathrm{a}} \text { motivating for } \\
\text { students to come to lectures }\end{array}$ & 74.0 & 65.4 & 68.6 & 24.0 & 30.8 & 23.5 & 2.0 & 3.8 & 7.8 \\
\hline Are better structured for presentations & 92.0 & 82.7 & 84.0 & 8.0 & 17.3 & 12.0 & 0.0 & 0.0 & 4.0 \\
\hline Are more $(\text { less })^{\mathrm{a}}$ beneficial for learning & 88.0 & 77.7 & 74.6 & 8.0 & 14.8 & 15.7 & 4.0 & 7.4 & 9.8 \\
\hline
\end{tabular}

${ }^{a}$ Words in brackets indicate the original terminology on the questionnaire. These questions were inversely rated. 
may help students better understand the material which in turn can be a source of motivation for lecture attendance. Indeed, previous studies that have experimented with PowerPoint have confirmed that the organisation and structure of the method can benefit students' learning (Holzl, 1997; Lowry, 1999).

The benefit for students' learning is perhaps the most vital issue in assessing electronic lecturing in education. In this preliminary study, about $75 \%$ of students $(88 \%$ of the first year students) felt that PowerPoint lectures were beneficial for their learning. To determine how beneficial PowerPoint lecturing was in learning, in reality, test results from an objective test, given after the first four lectures in Motor Learning module, were compared to the test results obtained by students in the previous year. In contrast to the hypothesised result, no significant differences between the two test results were found (mean $=19.5 / 30 \mathrm{SD}=3.8$ in $1997 / 98$ and mean $=19.9 / 30 \mathrm{SD}=3.4$ in 1998/99). Albeit these findings are in contrast to previous reports (Evans, 1998; Lowry, 1999), it should be emphasised that conditions leading to the test and the test given to the students were assumed to be identical. For example, the same lecturer delivered the same material. However, the experience and presentation style of the lecturer could have changed. Further, it is not impossible that students in the previous year had different academic abilities than those enrolled in the current year. Therefore, the assumption that the only difference between the 1997/1998 and the 1998/1999 cohort of students was the method of lecturing (i.e., overheads in 1997 vs. PowerPoint in 1998), may be questionable. Overall, the results of this study suggest that digital PowerPoint lecturing is perceived by the students as beneficial for learning, but the actual grades do not support this in this claim.

The second questionnaire used in this study provided an insight into the features that students appreciate in PowerPoint lectures. As illustrated in Table 2, the five most appreciated components of the method were: (1) variation of the fonts, (2) the use of illustrations, (3) a preference for light background, (4) the use of colours, and (5) the line-by-line projection of the lecture concepts. The finding that $72 \%$ of all respondents wanted to see PowerPoint adopted in all modules also reflects students' appreciation of the method. However, it is hard

Table 2

What students would like to see in digital PowerPoint lecturing. Based on 155 responses obtained from three classes (level 1 to 3) in the Sports Science Course

\begin{tabular}{llcc}
\hline Question & $\begin{array}{l}\text { Agree or strongly agree } \\
(\%)\end{array}$ & $\begin{array}{l}\text { Uncertain } \\
(\%)\end{array}$ & $\begin{array}{l}\text { Disagree or strongly disagree } \\
(\%)\end{array}$ \\
\hline Vary size and shape of fonts & 87.1 & 10.3 & 2.6 \\
Have more illustrations & 64.9 & 25.4 & 9.7 \\
Use light background with dark fonts & 62.7 & 26,8 & 10.5 \\
Use dark background with light fonts & 20.0 & 30.3 & 49.7 \\
Vary colors freely within the lecture & 51.0 & 29.1 & 19.9 \\
Use consistent color-scheme within the same & 33.5 & 36.2 & 30.3 \\
lecture & 3.9 & 7.3 & 88.8 \\
Use black and white presentations & 50.0 & 38.8 & 11.2 \\
Use line-by-line presentations & 4.6 & 8.5 & 86.9 \\
Abandon PowerPoint lecturing & 72.0 & 19.3 & 8.7 \\
Adopt PowerPoint lecturing in all modules & & & \\
\hline
\end{tabular}


to determine whether such a desire emerges from personal needs for better education or for better entertainment.

\section{Study II}

\subsection{Rationale and hypothesis}

Since the attention commanding feature of PowerPoint software (Holzl, 1997; Lowry, 1999) should result in better retention from one week to another, it was expected that students would obtain better overall grades on a series of three mock tests. Furthermore, since the elimination of note taking can help students to pay more attention to the lecture, it could be expected that PowerPoint in combination with the distribution of all lecture notes will result in improved retention. Therefore, it was hypothesised that PowerPoint lectures will yield better retention than overhead lectures. Further, it was presumed that PowerPoint lectures combined with the handing out of all the lecture notes will yield the best performance 1 week later on a ten-item multiple-choice mock test.

\subsection{Method}

Fifty-two second year students taking a compulsory 'Research Methods in Sports and Exercise' module took part in the study. Students were blind to the hypothesis without being subjected to deception. Initially, they were told that a series of mock tests would be given at the beginning of the lectures to measure their understanding of the previous lecture. They were instructed to maintain their usual studying habits. After each test, the grades along with the right answers, were provided to the students. At the end of the study, a graphical illustration of class performance under three lecturing conditions was provided to all students.

The study lasted for three consecutive weeks from the start of semester two in 1999. Students had one 3-h lecture each week. The first lecture was delivered with an overhead projector and some use of the blackboard. The second and third lectures were delivered using PowerPoint 7.0 software installed on a Hi-Grade Notino MI3000 notebook from which it was projected to a screen via a portable remote controlled VGA projector. The only difference between the second and the third lectures was that at the beginning of the third lecture, students were given the lecture notes printed through PowerPoint. All the lectures lasted for $150 \mathrm{~min}$ divided into two halves by a break. The lecturing speed and number of questions stimulating interaction were maintained as consistent as possible across the three lectures. All the three lectures covered material of theoretical nature and a comparable number of key points were discussed in each lecture. One week after the lectures, a ten-item multiple-choice test was presented to students. All the three tests were presented with the aid of PowerPoint software. The tests were designed to be of approximately equal level of difficulty by forming the questions around the 10 most important concepts covered in the previous lectures. Students had $60 \mathrm{~s}$ to select the right answer to each question from five alternatives given and record their choice on a computer readable answer card. Ten seconds before the projection of the next 
question, students were asked to make a choice on the computer readable answer card if they had not done so already.

\subsection{Results and discussion}

The obtained data were analysed with repeated measures analysis of variance (ANOVA). Because some students did not attend one of the three lectures, the data analysis was restricted to those students who were present at all the three lectures. Consequently, the results obtained from 25 students writing all the three mock tests were included in the repeated measures ANOVA. The analysis yielded a statistically significant difference on the scores obtained on the three tests $(F(2,23)=33.0, p<0.001)$. The post-hoc comparisons, using Bonferroni corrected paired $t$-tests, revealed that the results were due to different grades obtained on the overheadbased and PowerPoint-based lectures. However, the fact that students were given the lecture notes before the start of the class did not make a difference (Fig. 1).

The findings from Study II indicate that PowerPoint lectures resulted in better performance on the multiple-choice test than overhead lectures. The fact that handouts did not yield a further increase in the students' performance may be attributed to a ceiling effect. Alternatively, the lecturing pace was comfortable and students could take notes without distraction to their attention.

Whilst these findings suggest that PowerPoint lecturing appears to benefit at least for retention they should be viewed cautiously. Many extraneous factors could have influenced these data. For example, there was no control over the amount of study students did between

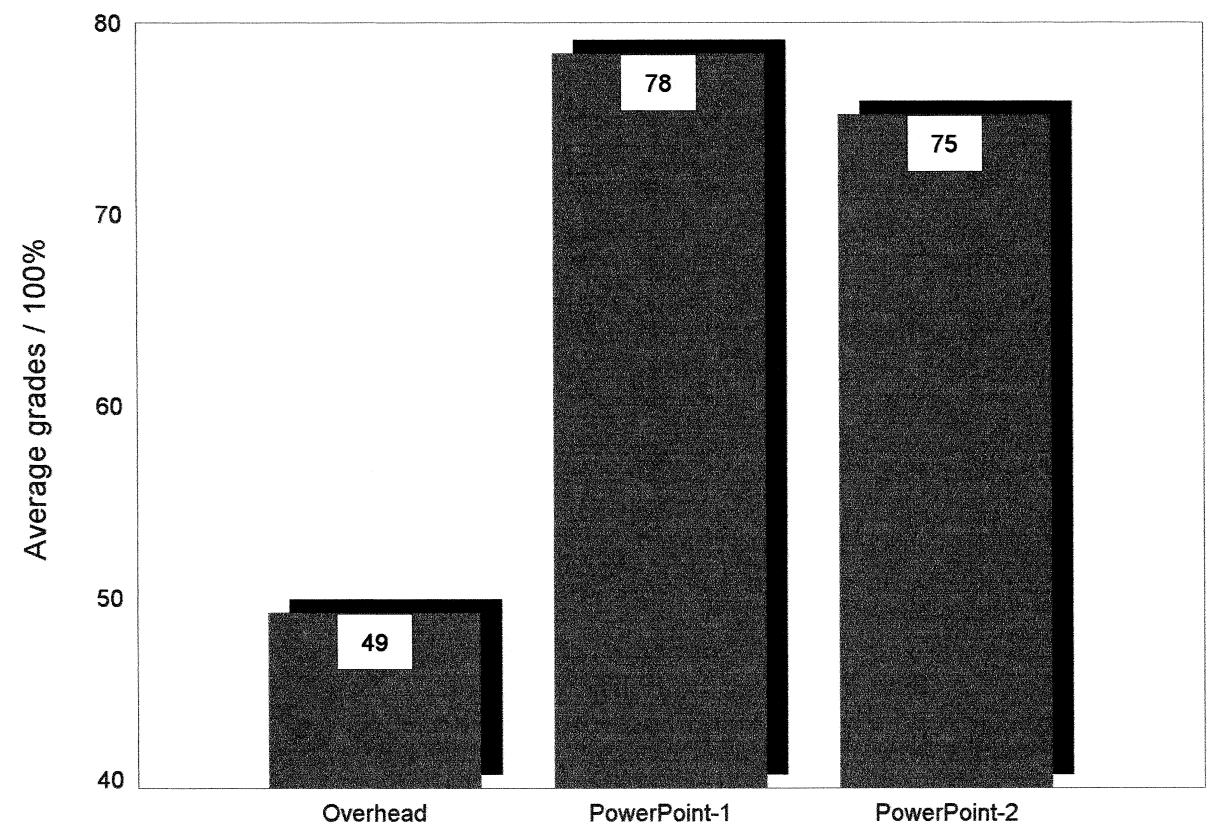

Fig. 1. Average grades obtained by 25 students, 1 week after a traditional overhead lecture, 1 week after a PowerPoint lecture and 1 week after a PowerPoint lecture supplemented with the handing out of the lecture notes. 
the lectures and the difficulty of the test question. Indeed, the mere number of questions and the fact that they revolved around the most extensively covered material in the previous lecture does not guarantee that they were equally difficult. The 'perceived level of difficulty' of the three lectures may have been different from the 'planned level of difficulty' which may have had an influence on test performance. To investigate the dilemma with preparation and further lecture difficulty, Study III was devised.

\section{Study III}

\subsection{Rationale and hypothesis}

Determining the efficiency of lecturing method simply based on students' results after one PowerPoint and one overhead lecture may not be very reliable. Practice effects may affect these results. Further, the problem of consistency, with regard to the level of difficulty of the lectures, remains unresolved. Another issue of concern in this type of investigation is that students' preparation for mock tests is unknown and no assumptions can be made in this regard. To address these issues of concern systematically, Study III was designed so that it could be assumed that the students prepared for the tests after both PowerPoint and overhead lectures. They were tested in practical sessions in which they could receive a real in-class quiz every week during semester two in 1999. Furthermore, since two groups were enrolled in these first year 'Sport and Exercise Psychology' practical sessions, one having the session on Mondays and one on Fridays, a counterbalanced design could be used. Obviously, under such conditions, learning and the role of the lecture medium in paving the road for learning, were tested rather than retention as in Study II.

\subsection{Method}

In Study III, two groups of students were tested twice, one week apart. The first group was a first year Sport Science cohort whilst the second group was a year one Combined Science (Biology and Sport) cohort. Both groups of students had to take the 20 Credit Point Units (CPU) Sport and Exercise Psychology Module in their second semester of their first year of studies. A 3-h practical session was delivered at weekly intervals as part of this Module. The Sport students attended the practical sessions on Mondays between $9.00 \mathrm{am}$ and $12.00 \mathrm{pm}$ whilst Combined Science students attended it on Fridays from $1.00 \mathrm{pm}$ to $4.00 \mathrm{pm}$. There were about 50 students in each group. Sixty-nine students completed the study by being present at both lectures (42 Sport Science students and 27 Combined Science students).

An overhead projector, a Viglen Pentium PC and a digital VGA projector were the technical equipment used in this study. Students in the two groups received an overhead and a PowerPoint lecture that represented the first $30 \mathrm{~min}$ of the practical session, in a counterbalanced order. The study ran over a period of two weeks, comprising two practical sessions in Sport and Exercise Psychology for both groups of participants. The Sport Science students received the PowerPoint based instruction on Monday week 1 whilst the Combined Science group received it on Friday week 2. The overhead based lectures were delivered on 
Friday week 1 for the Combined Science group and on Monday week 2 for the Sport Science group. One week after the respective lectures, students answered a ten-item multiple-choice test as in Study II on computer readable answer cards.

\subsection{Results and discussion}

The answer cards were computer-read and the grades were entered in Excel spreadsheets from where they were transferred into an SPSS (Statistical package for Social Sciences) statistical spreadsheet for subsequent analyses. Data were analysed with a group (Sport Science and Combined Science) by lecture (PowerPoint and overhead) repeated measures ANOVA. This analysis yielded a significant group main effect $(F(1,67)=18.0, p<0.001)$ indicating that the Sport Science students performed better on the two mock tests than Combined Science students and a significant group by lecture interaction $(F(1,67)=49.1, p<.0001)$. The further analyses of the interaction, with Bonferroni corrected $t$-tests, revealed that the Sport Science students performed better on the PowerPoint lecture-based test $(t(41)=6.53, p<0.001)$ whilst the Combined Science students performed better in the overhead lecture-based test $(t(26)=3.95, p<0.001)$. Consequently, both groups performed better on the test based on the lectures delivered in week 1 than on the test based on the lectures delivered in week 2 (Fig. 2).

These results show that lecture difficulty can make a difference. In Study III, using a counterbalanced research design, the superiority of the PowerPoint lectures in contrast to the traditional overhead lectures could not be demonstrated.

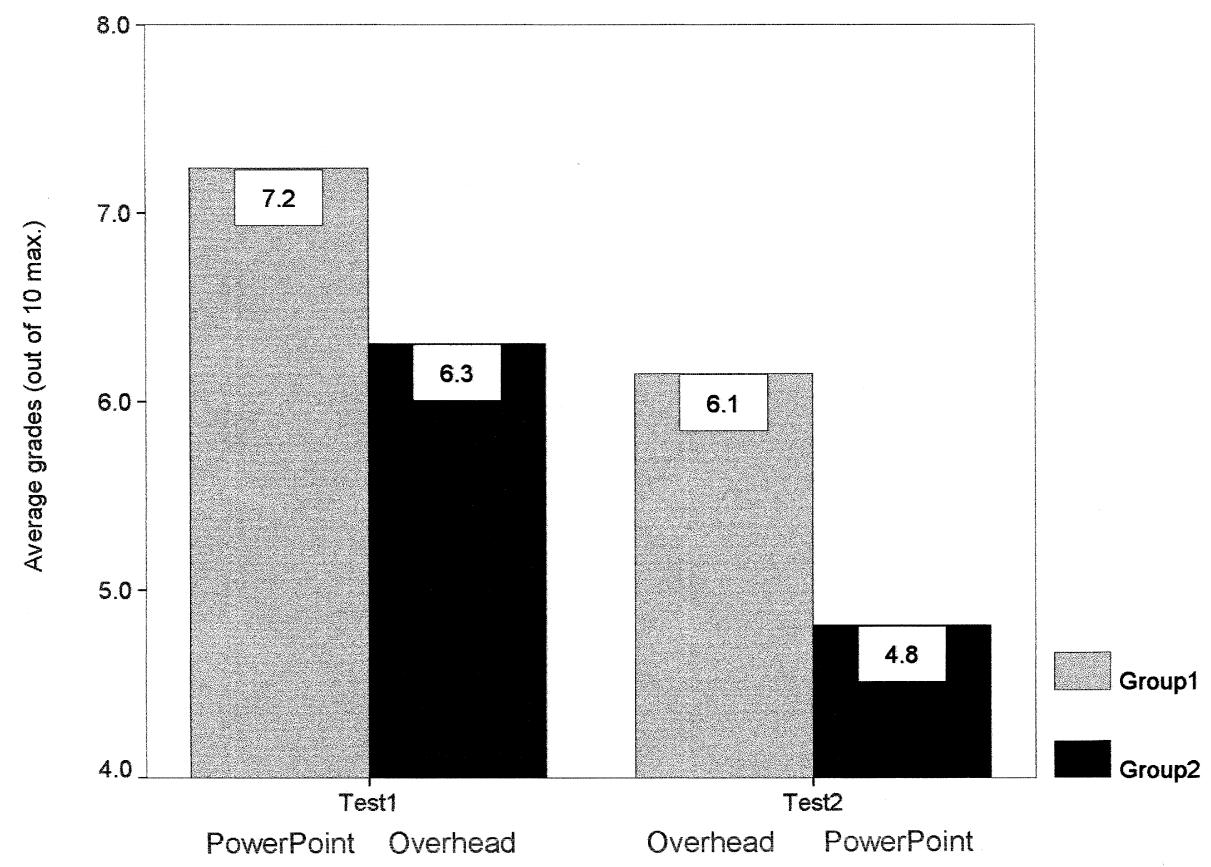

Fig. 2. Average grades obtained by two groups of students, 1 week after a traditional overhead lecture and 1-week following a PowerPoint lecture delivered to them in a counterbalanced order. 


\section{General discussion}

At the doorsteps of the new millennium, higher education faces a technological revolution that prompts changes in teaching and learning. These changes can be either transitional or transformational. The former are changes in methods of teaching whilst the latter are changes in aims, methods, and functional philosophies (Bergquist, 1992). In this research, three studies were performed to evaluate transitional changes in lecture delivery by replacing blackboard teaching with electronic lectures. Albeit there is some justification for using PowerPoint lectures (Holzl, 1997) and some evidence, based on students' learning, may lend support for such a transition (Evans, 1998; Lowry, 1999), the findings obtained in two out of three studies reported here do not support the need for an urgent motion for a transition from traditional to electronic lectures. Generally, it appears that PowerPoint lectures, at least in some circumstances, mainly add to the entertainment rather than to the education of the students.

At this time, the amount of research on PowerPoint lecturing is very scarce. With regard to students' performance, optimistic results were obtained only in Study II. In this study, mere retention was tested. If the number of lecture concepts were indeed comparable, as it was intended, then it can be claimed that PowerPoint lectures may be better for (memory) retention or recall than overhead lectures. This conjecture is in accord with Harknett and Cobane (1997) who suggested that some distinctive features of PowerPoint lectures, such as colour, could contribute to better recall. However, in Study II no control existed over the level of difficulty of the three lectures. Further, students' preparation could not be estimated. Could it be possible that PowerPoint lectures stimulate interest and, consequently stimulate independent learning? These questions remain unanswered. Hence, the findings from Study II should be viewed as tentative until more studies that are systematic are carried out.

In contrast to previous reports, the results emerging from Study I and Study III failed to support the notion that PowerPoint lectures benefit students' learning as based on assessment grades. In comparison to the study reported by Evans (1998), the academic subjects taught were relatively similar as neither Motor Learning nor Sport and Exercise Psychology are too far from General Psychology. The size of the groups was also comparable. Yet, the findings were different. The differences may be related to possible inequality of the groups in Study I here and in Evans' study. If a two-group counterbalanced design like the one in Study III here, is not adopted, the dilemma with equality of the groups could always be present (Lowry, 1999). This dilemma is accentuated by the results of Study III in which the two groups tested have shown different academic performance, regardless of the lecturing method used.

Consequently, if one group had received only the traditional lecture whereas the other only the electronic lecture, the wrong conclusion based on students' grades would have been deduced. Based on these results, it can be recommended that a semester-long counterbalanced design be adopted in the further exploration of the benefits of PowerPoint lecturing on students' learning.

Apart from possible benefits on recall, no significant advantages to PowerPoint lecturing were found in the series of three studies. The survey results obtained in Study I, however, clearly indicate that students like PowerPoint as a lecturing method. Their preference for PowerPoint lectures, in contrast to their beliefs, is not accompanied by better academic performance. It appears that the novelty of PowerPoint is entertaining for students. These 
results only pertain to instruction where the delivery system places emphasis on 'process' with unspecified objectives (Heywood-Everett, 1991). Indeed, in the series of studies reported here, only the mere switch between the traditional and the novel electronic method was tested. This switch does not result in significantly better learning. However, such findings do not invalidate the thought that PowerPoint can benefit learning in specific circumstances, where the objectives are better achieved with electronic lecturing.

The challenge in the new millennium is not to entertain students, that can be achieved with PowerPoint as based on the results from Study I, but to improve or to facilitate learning (Sipress, 1995). Based on the studies reported here, and in accord with theoretical conjectures (LeDuc, 1996), there is little or no advantage in lectures where simply the process or the delivery medium is changed.

\section{Conclusions}

The mere replacement of the blackboard with electronic lectures delivered via PowerPoint does not result in superior academic performance in Psychology related modules as based on graded assessments. It appears, however, that PowerPoint lecture may benefit recall (or perhaps recognition) from memory. PowerPoint could be useful in specific instruction where dynamic models, animation, and variation of colour may definitively help in the better illustration of the key concepts. Thus PowerPoint should not be viewed as a replacement for the blackboard, but rather as an efficient auxiliary medium, that can improve learning. Otherwise, PowerPoint will only entertain, rather than educate, students.

\section{References}

Bergquist, W. (1992). The four cultures of the Academy. San Francisco: Jossey-Bass.

Evans, L. (1998). Preliminary study: lectures versus PowerPoint. 4.0 http://www.kcmetro.cc.mo.us/longview/ lect_ppt.HTM.

Harknett, R. J., \& Cobane, C. T. (1997). Introducing instructional technology to international relations. Political Science and Politics, 30, 496-500.

Heywood-everett, G. (1991). Computer instruction in the classroom context. Early Child Development and Care, 69, 71-79.

Holzl, J. (1997). Twelve tips for effective PowerPoint presentations for the technologically challenged. Medical Teacher, 19, 175-179.

LeDuc, A. L. (1996). What is truly important about information technology in higher education. Journal of Computing in Higher Education, 8, 124-139.

Lowry, R. B. (1999). Electronic presentation of lectures - effect upon student performance. University Chemistry Education, 3(1), 18-21.

Sipress, M. (1995). Computer software in the undergraduate political science classroom. Paper presented at the 1995 Annual Meeting of the American Political Science Association in Chicago, ILL. 